\title{
Editorial
}

\section{Bioinformatics as an Increasingly Important Resource in Taxonomy, Phylogenetic and Diagnostics}

\begin{abstract}
This special Issue on Bioinformatics as an Increasingly Important Resource in Taxonomy, Phylogenetic and Diagnostics is an attempt to emphasize the importance of a multidisciplinary approach to these complex branches of Biology. Taxonomy and Systematics deal with classification of biological groups, mainly at the species level. Phylogeny tries to reconstruct the most likely history of species. Diagnostics is a discipline that takes advantage of all the classificatory knowledge to individuate rapidly and precisely the species of interest, especially in clinical environments. The core object of all these activities is the species on which a debate exists to determine whether it is as mere conventional categories or as real structure and, more generally, to produce a largely shared species concept. This concept is even more complex in Microbiology, a relatively young science which has often taken concepts, definitions and operations from general Biology, although we are now aware of the deep difference between the genetic determinants in Microbiology and in the rest of Biology. The presence of massive horizontal gene transfer, the short time interval between mutation and selection, the fact that many microbes are haploid and then all mutations are immediately selectable, the possibility to reproduce asexually or with a sexuality which often trespasses the possible boundaries of the species, are some of the many problems the microbiologist deal with in their everyday struggle to catalog microbial biodiversity and to understand its structure.
\end{abstract}

Every classification relies on a theory, or is a theory itself and gives the framework for the elaboration of new theories. The classification is therefore not important only for its mere practical outcomes, but rather for its deep scientific meaning and for the possibility it offers for the advancement of Science.

This simple outline of the problems involved in classification gives an idea of the specialties necessary to gain deeper comprehension on microbial evolution and to define optimal systems for classification and identification.

This special issue opens with two papers on two basic concepts involved in every biological, but especially in microbial classification: the problems of the continuity of taxa and of the centrality of the type strains. Although Phylogenetic has long disregarded typology as a form of classification, the International Codes regulating microbial systematics are strongly based on a typological design, implying that indeed types exist and that they are meaningful. Cardinali and coworkers investigate on the statistical and bioinformatics tools to ascertain if discontinuities separate species, or if they should be considered mere nominalist sets. Their other article consider the problems of how to individuate the type strain that represent the species as a whole and warn on the problems deriving from non-central type strains.

A paper by Cletus W. Kurtzman, an eminent yeast taxonomist co-editor of the last two editions of the basilar book on yeast taxonomy The Yeasts: a taxonomic study, deals with the issue of using several genes to classify microorganisms, in this case yeast. This topic is crucial from a practical point of view and has important relapses on another theoretical and highly debated issue on the possibility to reduce organisms to their genes. The Kurtzman paper explains quite clearly how the use of several marker genes produces several important outputs and how to deal with these sequences.

The most advanced application of this practical approach is the barcoding project treated in detail by two papers by Lewis and coworkers and by Robert and collaborators. With different approaches, both papers show a series of innovative and interesting bioinformatics analyses to individuate the best possible candidate as marker genes for classification and identification. The obtained results are quite stunning and will undoubtedly have practical implication for microbial identification in the future.

As stated above, the several routes of microbial gene flow are one of the most complex issues to understand their genetics and therefore their classification and phylogenetic history. A paper by Fani and others deals with the aspects of the integration of plasmids in bacterial chromosomes.

A paper by the Brandao group deals with the possibilities offered by the analysis of metabolomics data. This is a reminder that microbes are not only genes and that, after all, they are important particularly for their metabolic activity. This article is the only one of this Special Issue to deal with the analysis of continuous quantitative data rather than discontinuous and categorical as the DNA sequences.

The article is closed by two articles presenting very useful practical tools. Nilsson and others introduce the UNITE database designed to help ecologists in the endeavoring task of identifying the environmental fungi, which are likely to represent some $98 \%$ of the whole fungal biodiversity. The last paper by Robert and collaborators describes BioloMICS, a large and multipurpose software application for storage, management, publication and statistical analyses of any biological, taxonomical, physiological, molecular and ecological data. 
This Special Issue has been conceived as a trip from theory to practice in the universe of the bioinformatics applied to Taxonomy, Phylogenetics and diagnostics. Most of the articles deal with yeasts not only for their intrinsic importance, but rather for they prominent role as model organism and for the advancement that yeast taxonomy and systematics have reached in the last half century.

As guest editors, we are indebted to all authors for their contributions and for the effort to stay in the allotted spaces and times.

\section{Gianluigi Cardinali}

(Guest Editor)

Department of Applied Biology

Microbiology Division

University of Perugia

Italy

E-mail: gianlu@unipg.it
Vincent Robert

(Guest Editor)

CBS-KNAW Fungal Biodiversity Centre

Utrecht

The Netherlands

E-mail: v.robert@cbs.knaw.nl

(C) Cardinali and Robert; Licensee Bentham Open.

This is an open access article licensed under the terms of the Creative Commons Attribution Non-Commercial License (http://creativecommons.org/licenses/by-nc/ 3.0/) which permits unrestricted, non-commercial use, distribution and reproduction in any medium, provided the work is properly cited. 